\title{
APPLICATION OF THE E - LAYER MODEL FOR SOLVING THE PROBLEMS OF PARAMETRIC ESTIMATION IN MEASURING DEVICES
}

\author{
Alexey Yakunin* and Larisa Suchkova \\ Altai State Technical University, 656038, Barnaul, Russia
}

\begin{abstract}
The article proposes a technique for estimating the maximum possible errors in measuring the parameters of the signals observed against a background of interference that do not exhibit statistical properties on the measurement interval and of which only the region of their possible change is known. This region is a layer within which the interference -free signal can vary due to the variation in the values of the parameters of the model function describing it. The technique is also proposed that makes it possible to estimate the measurement error of the monitored parameter for a specific implementation of the signal described by the proposed model with a confidence probability close to unity. An example of the use of the offered techniques for estimating the coordinate of a light stain created by a point source is shown. The offered approach is actual, when each measurement represents special worth and there is no possibility to improve the measurement result by averaging over the set of signal realizations.
\end{abstract}

\section{General definition of the problem}

At calculation, designing and use of control and measuring devices the estimation of their potentially achievable characteristics of accuracy and measurement errors is of great importance, as defines the basic metrological characteristics of devices and the demands shown to their hardware representation [1]. In particular, level of internal noise and maximum deviations from rating values of electronic components, capacity of analogue-to-digital converters, capacity of a memory for a data storage, type of variables used for a data handling.

For an estimation of errors of measurement mainly statistical approach now is used [2-7]. Its application is justified, when value of parameter inspected by the measuring device is selected from a quasi-determined signal containing additive stationary ergodic noise. However not for all factors influencing a controllable signal their laws of distribution can be set. Moreover, sometimes the informative signal is presented by unique realization of nonstationary acyclic process with the limited number of references that excludes a possibility of use of classical statistical methods of its processing. Therefore in cases when in the course of measurement statistical properties of controllable process are not exhibited, the possibility of carrying out of repeated measurement is absent, each separate measurement has special value and it is necessary to define a range of possible values of outcome of

* Corresponding author: yakunin@agtu.secna.ru 
measurement, more expediently to find an interval estimation of controllable parameter and potential accuracy of such estimation with application of methods of the interval analysis [810]. Such methods are well developed with reference to problems of an estimation of errors of outcomes of joint and cumulative measurements in various applied areas $[11,12]$. Generally the vector of required controlled parameters $\lambda$ with dimension $m$ in such cases defined by results of measurements $\left(x_{i}, y_{i}\right)$, connected by expressions

$$
y_{i}=F\left(x_{i}, \lambda\right) ; x_{i}, y_{i} \in X ; \lambda \in \Lambda \text {, }
$$

where $i=1, \ldots, n$, and $y_{i}$ and $x_{i}$ are measured with accuracy $\varepsilon \in X, X$ and $\Lambda$ - spaces of accordingly measured and controllable values.

However in modern intellectual informational-measuring systems often it is necessary to deal not with a final population of outcomes of measurements, but with continual and in some cases also with many-dimensional multiparameter signals. It can be, for example, twodimensional optical signals varying in time, or wavelet-transformations of acoustic signals. A part from parameters of such signals are form parameters, a part - support parameters, and only small part - the informational parameters defining estimation of required value.

For determination of an interval estimation of errors of measurement of parameters in such cases in works [13-15] it is offered to use a method based on application of model of $\varepsilon$ - layer for exposition of signals with high degree of a priori uncertainty about conditions of their observation. We will consider at first the general approach to application of this model for calculation of potential accuracy and errors of measurement of measuring devices, and then - its application for a solution of a concrete practical problem.

\section{E - layer model and its using for solving the problem of estimation of potential accuracy of measuring equipment}

According to model of $\varepsilon$ - layer the signal containing controllable parameter is represented in the form of superposition of quasi-determined component (modelling function) of the useful signal and some component including random casual noise and interference, and also other deviations of a real signal from its description by the chosen modelling function. This component in all range of definition of a signal is described by an arbitrary class continuous or even explosive and not submitting to conditions of Lipschitz functions. On this function unique restriction is superimposed. It consists in that all values in a signal range of definition laid on an interval $\left[-\varepsilon^{-} \varepsilon^{+}\right]$, where $\varepsilon^{-}$and $\varepsilon^{+}$- some positive values, which estimations, as a rule, much less than maximum value of the module quasi-determined component.

Let's suppose for simplicity of reasoning, that all describing a quasi-determined component of model of a signal the set of parameters consists only of one controllable parameter $\lambda \in \Lambda$ concerning which the interval estimation is searched, and all remaining parameters $\xi \in \Xi$ among which can be both form and support parameters, and other informative parameters which also can be subject to measurement. Then for any arbitrary value $\lambda_{0} \in \Lambda$ according to $\varepsilon$ - layer model it is possible to note, that the following relation is fulfilled:

$$
S\left(r, \lambda_{o}, \xi\right)-\varepsilon<S\left(r, \lambda_{e}, \xi\right)<S\left(r, \lambda_{o}, \xi\right)+\varepsilon,
$$

where $\lambda_{e}$ - estimation of controllable parameter, $S\left(r, \lambda_{x}, \xi\right)$ - quasi-determined component of a signal, $r \in R$ - vector in space of coordinates $R$ on which function $S\left(r, \lambda_{e}, \xi\right)$ is defined. This vector can include in itself of three coordinates in three-dimensional space and the fourth coordinate - time. 
Generally instead of the fixed interval $[-\varepsilon, \varepsilon]$ (a layer of the fixed thickness) it is possible to use interval function $\left[-\varepsilon^{-}(r), \varepsilon^{+}(r)\right]$ if in the field of signal observation there are sections with various degree of accuracy of its exposition. Obviously, the lower the level of noise and interference and the less inaccuracy of the correspondence of the model function to the real signal, the thinner will be the thickness of the layer.

Let's consider now as by means of $\varepsilon$ - layer model it is possible to find potential accuracy of the measuring device on which input signal described by the equation (2) is fed. Potential accuracy at calculation and designing of control and measuring devices is of great importance [1]. Potential accuracy shows, what minimum possible errors of measurement can be received for the given aspect of a signal by means of the most perfect algorithm of its handling, but at the same time at the most unfavorable cases of its realization. And in this sense potential accuracy is its minimax estimation. Besides, being an interval estimation, it sets a resolving power of the measuring device, and finally defines its dynamic range and volume of the information received at measurement $[16,17]$. Indirectly potential accuracy allows to estimate also possibilities of the further perfection of performances of accuracy of developed equipment. By definition, the interval estimation $\left[\lambda_{-}, \lambda_{+}\right]$is a domain of definition with a given confidence probability of the point estimation $\lambda_{e}$ of the controlled parameter $\lambda$, taking in concrete realization of the observed signal having parameter value $\lambda_{o}$. According to a model of $\varepsilon$-layer, such estimation should satisfy to an inequality (2) and its confidence probability aspires to unity. Then $\Delta \lambda=\lambda_{+}-\lambda_{\text {- will define an }}$ absolute error of measurement of parameter $\lambda$ for its true value $\lambda_{o}$. Generally this error will be function of all parameters of a signal entering into the determined component. For true value of parameter $\lambda$, equal $\lambda_{o}$, it is possible to define the greatest possible value of this error as

$$
\Delta_{\lambda}\left(\lambda_{0}\right)=\max _{\xi \subset \Xi}\left(\lambda_{+}\left(\lambda_{o}, \xi\right)-\lambda_{-}\left(\lambda_{o}, \xi\right)\right)
$$

and the maximum error of an estimation of parameter $\lambda$ in all range of its modification can be defined as

$$
\Delta_{\lambda \cdot \max }=\max _{\lambda o \subset \Lambda}\left(\Delta_{\lambda}\left(\lambda_{0}\right)\right)
$$

Expression (4) represents an upper bound of an estimation of an error of measurement of controllable parameter which can be received as a result of handling of signal $S(r, \lambda, \xi)$. Value of an error set by this expression is defined only by properties of a signal, instead of algorithm of its handling. Therefore it cannot be reduced at use even the most perfect algorithm and will define magnitude of potential accuracy which can be reached for the given signal.

Comparing expression (3) with expression (2), it is easy to be convinced, that it is possible to present it in an aspect

$$
\Delta_{\lambda}\left(\lambda_{0}\right)=\max _{\xi \subset \Xi} \lambda_{+}\left(\lambda_{o}, \xi\right)-\min _{\xi \subset \Xi} \lambda_{-}\left(\lambda_{o}, \xi\right)
$$

where value $\lambda_{+}$and $\lambda_{-}$are radicals of a solution of the equations

and

$$
S\left(r, \lambda_{e}, \xi\right)-S\left(r, \lambda_{o}, \xi\right)=-\varepsilon^{-}(r) \text { for } S\left(r, \lambda_{e}, \xi\right)-S\left(r, \lambda_{o}, \xi\right)<\varepsilon^{+}(r)
$$

$$
\mathrm{S}\left(r, \lambda_{e}, \xi\right)-S\left(r, \lambda_{0}, \xi\right)=\varepsilon^{+}(r) \text { for } S\left(r, \lambda_{e}, \xi\right)-S\left(r, \lambda_{o}, \xi\right)>-\varepsilon^{-}(r)
$$

Thus from the discovered values greatest be assigned for $\lambda_{+}$, and smaller for $\lambda_{-}$.

If the thickness of the layer is sufficiently small, then the deviations $\Delta \lambda$ and $\Delta \xi$ of the signal parameters $\lambda$ and $\xi$ from their true values $\lambda_{o}$ and $\xi_{o}$ must also be small so that the signal 
realization does not exceed the limits specified by the $\varepsilon$-layer. Then it is possible to take advantage of linear approximation of function $S(r, \lambda, \xi)$ in a point neighbourhood $\left(\lambda_{o}, \xi_{o}\right)$, having presented the left parts of the equations (6) and (7) in the form:

$$
S\left(r, \lambda_{e}, \xi\right)-S\left(r, \lambda_{o}, \xi\right)=\left.\frac{d}{d \lambda} S(r, \lambda, \xi)\right|_{\lambda=\lambda o, \xi=\xi o} \cdot \Delta \lambda+\left.\sum_{i=1}^{n} \frac{d}{d \xi} S(r, \lambda, \xi)\right|_{\lambda=\lambda o, \xi=\xi o} \cdot \Delta \xi_{i}(
$$

Such representation allows to linearize the equations (6) and (7), that essentially simplifies their solution.

Thus, it is necessary for determination of potential accuracy at first with application of expression (8) or it is direct from (6) and (7) to discover boundaries of an interval estimation $\left[\lambda_{-}, \lambda_{+}\right]$, and already then from (3) and (4) to search for extreme value of a difference of these boundaries.

\section{Using the E - Layer Model to Estimate Interval Errors of Measurement for a of a Signal Realization}

From the statistical theory it is known, that in cases when conditions of a stationarity and an ergodicity of observable process are not observed, there can be so-called superefficient estimations when the confidential interval of controllable parameter for concrete realization can appear an arbitrarily small and even to become equal to zero [3,4]. It at all does not contradict expression (4) which sets only greatest possible value of an interval estimation for the most unfavorable case, instead of its value for concrete realization of a signal which will be always less or equally magnitudes finding from expression (4).

Let we have such concrete realization $S_{x}(r, \lambda, \xi)$. As this realization should be in limits $\varepsilon$ - layer, the quasi-determined component of a signal for any value $\mathrm{r}$ cannot appear more than $S(r, \lambda, \xi)+\varepsilon^{-}(r)$ and less than $S(r, \lambda, \xi)-\varepsilon^{+}(r)$, that is

$$
S\left(r, \lambda_{o}, \xi\right)-\varepsilon^{+}(r)<S_{x}\left(r, \lambda_{e}, \xi\right)<S\left(r, \lambda_{o}, \xi\right)+\varepsilon^{-}(r)
$$

It is clear from the comparison of (9) and (2) that the process of finding the measurement error for a particular implementation will not differ much from the case of calculating the potential accuracy, and accordingly, we can use the same algorithm to find the measurement error. The only, but rather important, difference is the substitution in (2), as a rule, of a smooth model function $S\left(r, \lambda_{0}, \xi\right)$ by a concrete realization of the signal $S\left(r, \lambda_{e}, \xi\right)$, representing the sum describing a quasi-determined component of modelling with the inherent noise component and the model inaccuracy lying within the $\varepsilon$-layer defined by the boundaries $\varepsilon+$ (r) and $-\varepsilon-(r)$. Such a function, unlike the model one, can contain a lot of irregularities, overfalls, protuberances and hollows. This difference also allows to receive for concrete realizations of an error, essentially smaller the errors set by potential accuracy.

For acknowledgement of the given fact we will consider the elementary one-dimensional case when the quasi-determined signal component represents a constant, and $\varepsilon$ - layer is constant and symmetric, that is $\varepsilon^{+}(r)=\varepsilon^{-}(r)=\varepsilon$, and $S\left(r, \lambda_{o}, \xi\right)=\lambda$. For such a signal, depending on its physical nature, $r$ can be time, or one of the spatial coordinates. It is obvious, that for such signal the parameter $\lambda$ can accept values only from an interval $\left[\lambda_{-}, \lambda_{+}\right]$, and the maximum error of its measurement $\Delta \lambda$ can reach values $2 \varepsilon$.

Such error will be observed when the real signal in all area of the definition has the fixed value that is improbable, but, nevertheless, probably. Besides the considered model assumes, that each realization of a signal is unique and to improve outcome of observation by its average on a series of measurements it is impossible, as the interference accompanying a 
signal throughout all series of measurements can not exhibit statistical properties and remain invariable. At the same time such improbable situations, when $\Delta \lambda=0$ are possible also. The example of such situation is shown in Figure $1 a$ when on an interval of observation a signal undergoes a jump from the lower boundary to the upper bound $\varepsilon$ - layer, varying on magnitude $2 \varepsilon$.

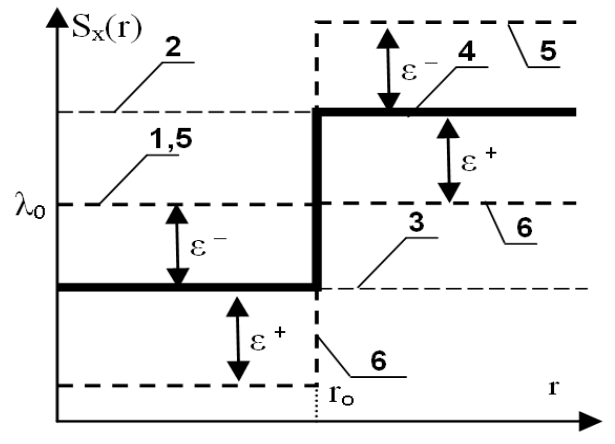

a

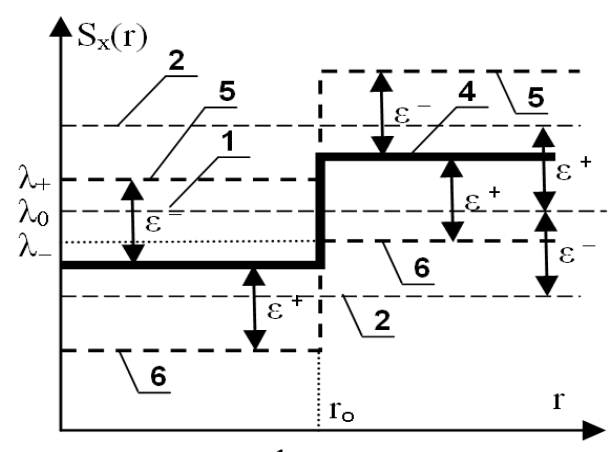

$\mathrm{b}$

Fig. 1. Determination of an interval estimation for concrete realizations of signals. 1 - the modelling function corresponding to true value of $\lambda_{0}$ observable signals; 2 - an upper bound of $\varepsilon$ - layer for modelling function with parameter $\lambda_{0} ; 3$ - the lower boundary of $\varepsilon$ - layer for modelling function with parameter $\lambda_{0} ; 4$ - observable realization of signal $S_{x}(r) ; 5$ - an upper bound of values of modelling function for an observable signal; 6 - the lower boundary of values of modelling function for an observable signal. a) rise of a signal is equal to a full width $\varepsilon$ - layer . b) rise of a signal is less than a full width $\varepsilon$ - layer

For this case $\lambda_{+}=\lambda_{-}=\lambda_{0}$, that is at such realization it is possible to restore absolutely precisely true value of controllable parameter, when $\lambda_{e}=\lambda_{0}$. However, if the swing width is less than the total thickness of the $\varepsilon$-layer, the confidence interval will have a finite length equal to the difference between the total thickness of the $\varepsilon$-layer and the value of the drop (Figure $1 b$ ). We will consider now an example of application of the offered methods of determination of interval estimations not for abstract, and for the real optical signal projected on an input of the one-line converter of the image of the device for the monitoring linear movements.

\section{Computational experiment for modeling the estimation procedure of the interval error as applied to the passive optical positioning system}

Optoelectronic devices are widely applied in measuring technique to the solution of the most various problems, including for positioning of remote objects by non-contact methods [1821]. For this purpose, a light source is fixed at the monitoring object or a light spot is formed on it. The light source or a spot is projected on a surface of a photodetector sensitive to movements or on the image converter in an electric signal (for example, CCD $[22,23]$ ) and a position of a projection of a light source define an object position. Obviously, accuracy of positioning will be defined first of all by quality of formation of an optical signal an objective and presence in a controllable scene of the image or on a section between object and an optical system of additional installations, presence of background illumination and other factors, to consider which difficult or even it is impossible.

To demonstrate the application of the $\varepsilon$ - layer model to estimate the error caused by these dominant factors, consider the case where the image of the luminous source placed on the 
object is projected onto the surface of a linear CCD photodetector. If to assume, that a source is dot, that in this case it is quite admissible, the signal shape on certain wave length and under using an ideal (no aberration) system will be described by a Bessel's function of first order $\mathrm{J}_{1}(\mathrm{kr})$ with a scale factor $k$, proportional to diameter of an entrance pupil of objective $D_{0}$ and inversely proportional to a focal length of objective $F_{o}$ as for enough remote installations the image plane will coincide with a focal plane [24]. As the optical signal will be perceived by a photodetector only over the range lengths of waves of its spectral response the useful two-dimensional signal will be equal to integral from $J_{1}(\mathrm{kr})$ on the given range. Since in semiconductor photodetectors the upper limit of the spectral range is at least twice as high as the lower, the result is a signal that is close in shape to a gaussian:

$$
E(x) \equiv E\left(x, x_{0}, R\right)=E_{0} \cdot e^{-\frac{\left(x-x_{0}\right)^{2}}{R^{2}}}
$$

where $E_{0}$ - the maximum level of light exposure created by a light source on a surface of a photodetector, $x$ - cartesian coordinate, $x_{0}$ - coordinate of the center of a source image on a photodetector surface, and $R$ - some parameter, proportional to effective radius of Airy scattering circle and in addition considering not ideality of an optical system [24].

As it is supposed, that concerning other components of an optical signal any information is absent, we will present them in the form of surrounding a signal $\varepsilon$-layer. Thus positive values will be created by the additional reflecting light installations which are in a zone of the control, and negative - by shading installations. As an instance of such installations the clouds recoating a laser beam at sounding of an aerosphere can serve, for example. Then the general model of an optical signal can be written down in an aspect

$$
E^{\prime}\left(x, R, x_{0}\right)=E\left(x, R, x_{0}\right)+\varepsilon(x),
$$

where $-\varepsilon^{-}(x) \leq \varepsilon(x) \leq \varepsilon^{+}(x)$.

From here, according to (8), it is possible to note, that the estimations of any called by errors of parametres of a signal of its deviation from an initial aspect will be equal:

$$
\Delta \mathrm{E}(\mathrm{x})=\mathrm{E}^{\prime}(\mathrm{x})-\mathrm{E}(\mathrm{x})=\frac{d}{d x_{0}} E\left(x, R, x_{0}\right) \cdot \Delta x_{0}+\frac{d}{d R} E\left(x, R, x_{0}\right) \cdot \Delta R+\frac{d}{d E_{0}} E\left(x, R, x_{0}\right) \cdot \Delta E_{0},
$$

whete

$$
\begin{gathered}
\frac{d E}{d E_{0}}=e^{-\frac{\left(x-x_{0}\right)^{2}}{R^{2}}} ; \\
\frac{d E}{d x_{0}}=2 E_{0} \cdot \frac{x-x_{0}}{R^{2}} e^{-\frac{\left(x-x_{0}\right)^{2}}{R^{2}}} ; \\
\frac{d E}{d x_{0}}=2 E_{0} \cdot \frac{\left(x-x_{0}\right)^{2}}{R^{3}} e^{-\frac{\left(x-x_{0}\right)^{2}}{R^{2}}} .
\end{gathered}
$$

Then, solving the equations

$$
\begin{gathered}
\Delta E(x)-\varepsilon^{+}(x)=0 \text { with } \Delta E(x) \geq-\varepsilon^{-}(x) \\
\Delta E(x)-\varepsilon^{-}(x)=0 \text { with } \Delta E(x) \leq \varepsilon^{+}(x)
\end{gathered}
$$


concerning deviations of any of parameters of function $E\left(x, R, x_{0}\right)$ at the fixed values of remaining parameters in the field of their possible modification, and then, discovering for them the greatest probable values, it is possible for that to discover the greatest probable errors of measurement of all parameters entering into an optical signal.

Examples of calculation of the greatest possible and actual values of errors for the considered model of a signal are shown in Figures 2-7. For calculations the normalized values of parameters $E_{0}=1, R=1$ and $X_{0}=0$ were set, and $\varepsilon$ - layer relied symmetric and invariable on a thickness which has been chosen equal 0.1. For such a thickness of the layer, the linear approximation (12) turned out to be unjustified and a solution for the initial model of the quasi-deficient component given by expression (9) was used. If variations of amplitude of signal $\mathrm{E}_{0}$ and effective radius are absent, the greatest possible error of definition of coordinate of light stain $\mathrm{X}_{0}$ makes 0.1168 (Figure 2) and if thus the effective radius increases by $10 \%$ (for example, at the expense of an objective defocusing) this error will decrease to 0.042 (Figure 3).

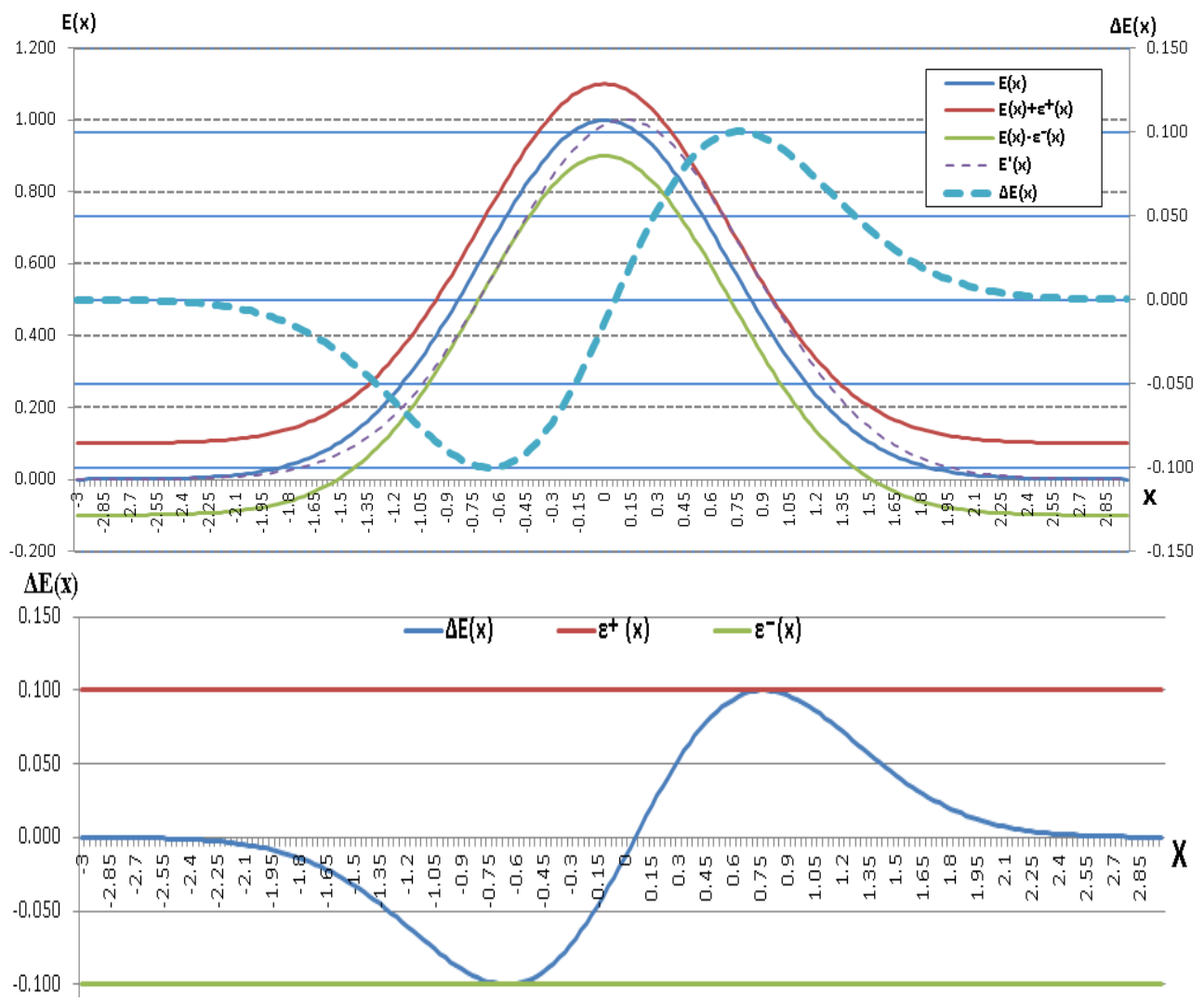

Fig. 2. Initial signal $E(x)$, upper and lower boundaries of $\varepsilon-$ layer $E(x)+\varepsilon^{+}$and $\mathrm{E}(\mathrm{x})-\varepsilon^{-}$, the greatest possible variation of signal $E^{\prime}(x)$ on parameter $\mathrm{x} 0$ and corresponding to this variation of the signal deviation $\Delta E(x)$ from its original form. 

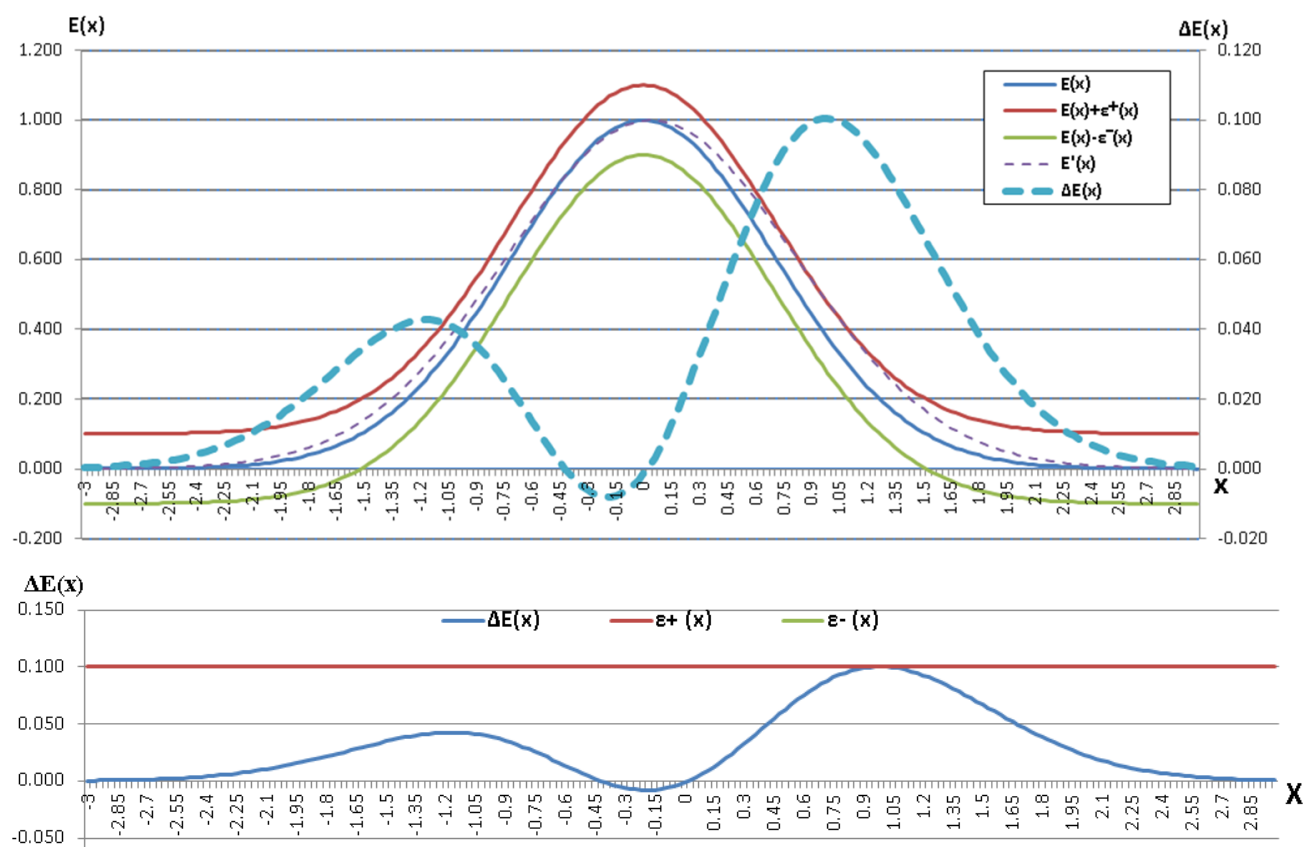

Fig. 3. Outcomes of evaluations for a case of the greatest possible variation of parameter $\Delta x$, at increasing of effective radius $R$ on 0.1 and invariable value of parameter $E_{0}$.

On the other hand, if coordinate of a light stain to leave invariable, but thus to increase amplitude $E_{0}$ of modelling function $E(x)$ to its greatest possible value within the set thickness $\varepsilon$ - layer, that is to 1.1 the maximum error of an estimation of parameter $R$ will appear equal -0.16 . Thus, the interval estimation for the effective radius will be sharply asymmetric with respect to $R$. The full correlation between boundary values of parameters $\Delta E_{0}$ and $\Delta R$ is presented in Table 1.

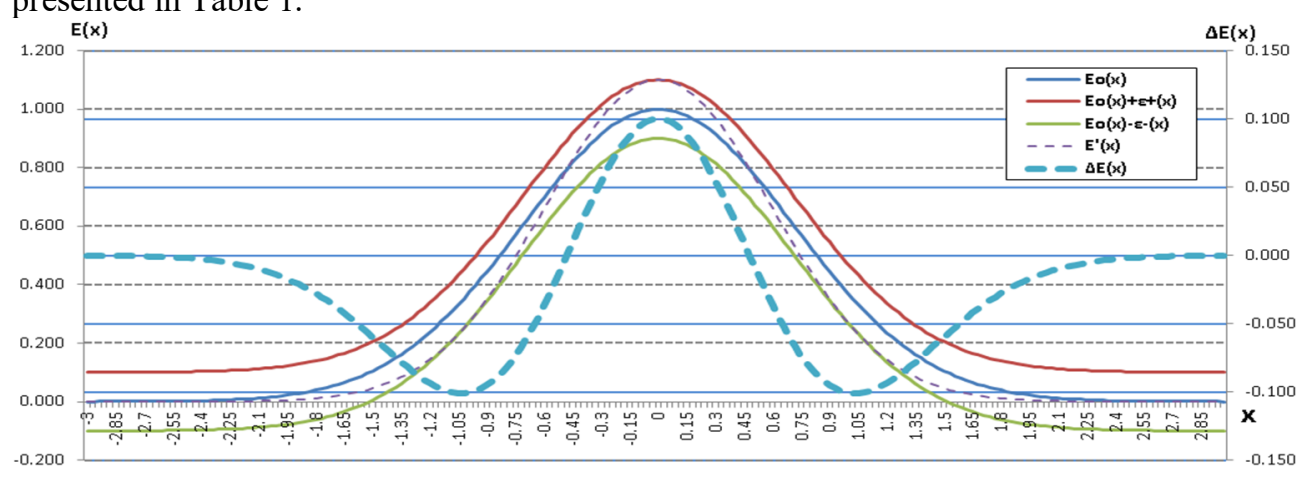

Fig. 4. Outcomes of evaluations for a case of the greatest possible positive variation $\Delta R$ of effective radius $R$ at invariable values of remaining parameters of a signal.

Table 1. The greatest possible variations of parameters of signal $E_{0}$ and $R$ under condition of absence of variations $X_{0}$.

\begin{tabular}{|r|c|r|c|}
\hline$\Delta E_{0}=$ & 0.00 & 0.10 & -0.1 \\
\hline$\Delta R=$ & {$[-0.1270,0.1461]$} & {$[-0.1583,0.0495]$} & {$[-0.0518,0.2101]$} \\
\hline
\end{tabular}


Unlike effective radius, the measurement error $\Delta x_{0}$ always will be symmetric, that is obviously visible from Figures 2, 4. As a whole the correlation between possible deviations of all three parameters of an optical signal is set by three-dimensional area. Since for these studies it is of interest to analyze only the maximum possible error in measuring the position of the light spot, the dependence of this error on the parameters $\mathrm{E}_{0}$ and $\mathrm{R}$ have been received. Outcomes of such calculations are presented in Figure 5 from which it is visible, that the maximum error will be observed at a choice $\Delta R=0$ and $\Delta E_{0}=0$ and is equal 0.1169 .

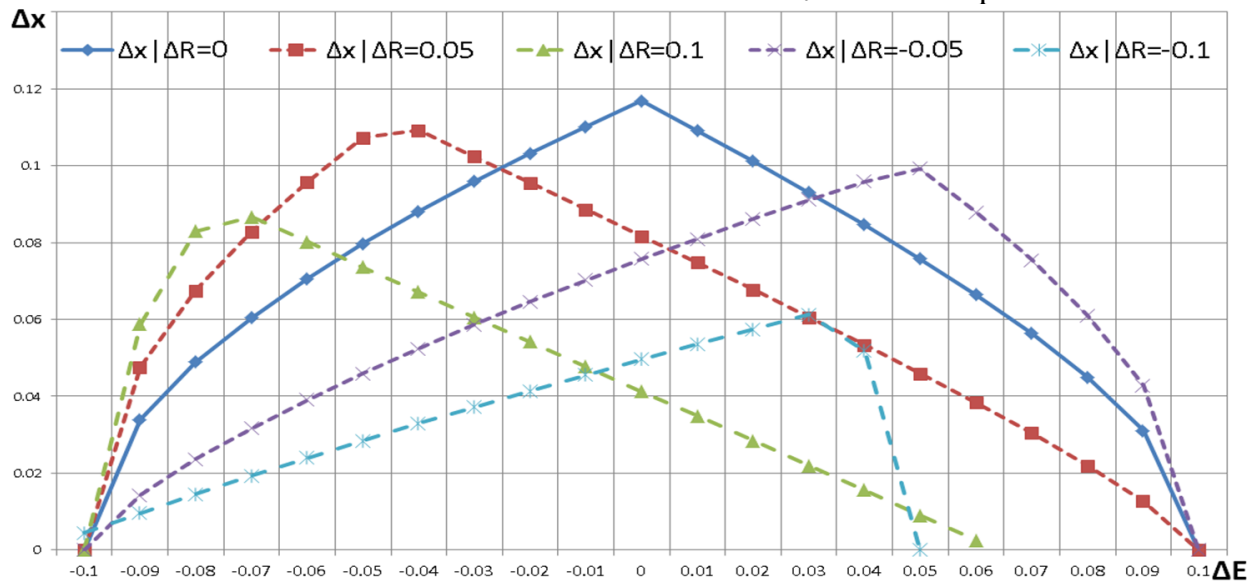

Fig. 5. Cross sections of the three-dimensional definition area of the variations of values of the signal parameters $E^{\prime}(x)$, provided it is in the region of the $\varepsilon$-layer of the original signal $E(x)$. The parts of the cross sections in the positive half-plane, mirror-symmetric with respect to the axis $\Delta E$ is shown.

At the same time the value of given estimation is maximally achievable and for concrete realizations it can appear essentially smaller, that is obviously visible from Figures 6, 7 .

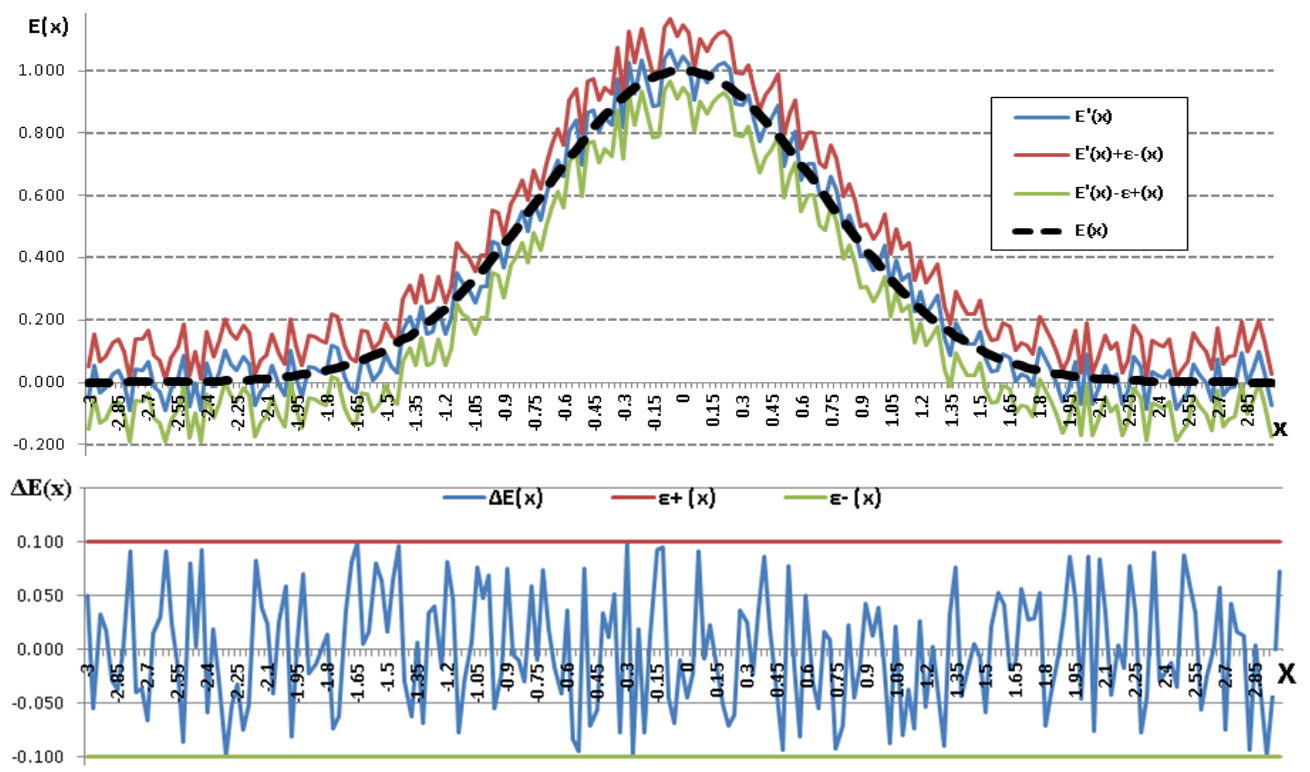

Fig. 6. Example of the implementation of the signal $E^{\prime}(x)$ and the possible boundaries $E^{\prime}(x)+\varepsilon^{-}(x)$ and $E^{\prime}(x)-\varepsilon^{+}(x)$ for the domain of the model function definition for the case of a nondeterministic component with a random process with a uniform distribution fun, given on the interval $[-\varepsilon,-\varepsilon]$. 


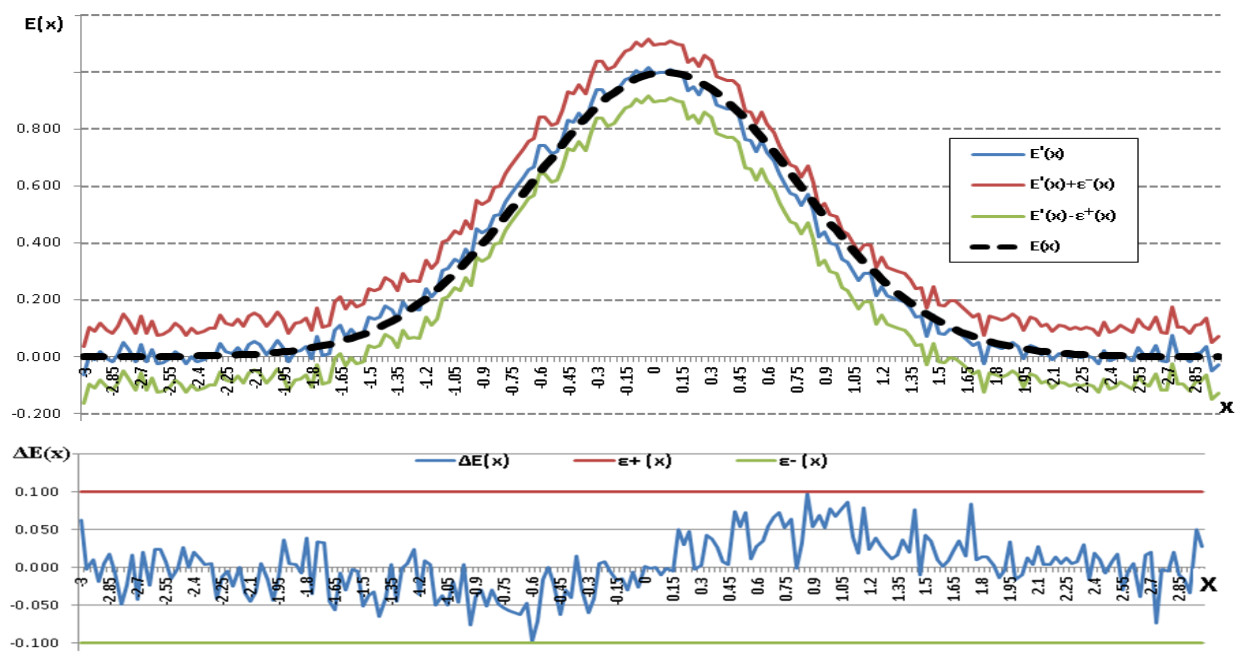

Fig. 7. Example of realization of signal $E^{\prime}(x)$ and possible boundaries $E^{\prime}(x)+\varepsilon^{-}(x)$ and $E^{\prime}(x)-\varepsilon^{+}(x)$ of a range of definition of modelling function for a case of representation of nondeterministic component as normal casual process with value of the variance equal $1 / 3 \varepsilon$ and an additional restriction of the density function of distribution by the interval $[-\varepsilon, \varepsilon]$.

For the signal realizations presented in Figures 6, 7 these values lay within 0.05 for the normal law of distribution and 0.008 for a rectangular distribution of signal variations. Thus as can be seen from these figures, an increase in the observation interval will lead to a decrease in the interval error estimation due to an increase in the probability of appearence the signal values close to the $\varepsilon$-layer boundaries. The maximum measurement error is reached at displacements $x_{0}$ by a value close to the value of the effective radius, when the model function touches the boundaries of the $\varepsilon$-layer. And the increase in the interval of observation does not allow to reduce the magnitude of this error, which is evident from Figures 6.7 However it is not necessary to forget, that the given approach was developed just for those cases when noise and other interference cannot be described casual process. If, for example, to suppose that there is a linearly varying background illumination in the observation zone, then the measurement error $x_{0}$ will increase at once, that obviously illustrates Figure 8.

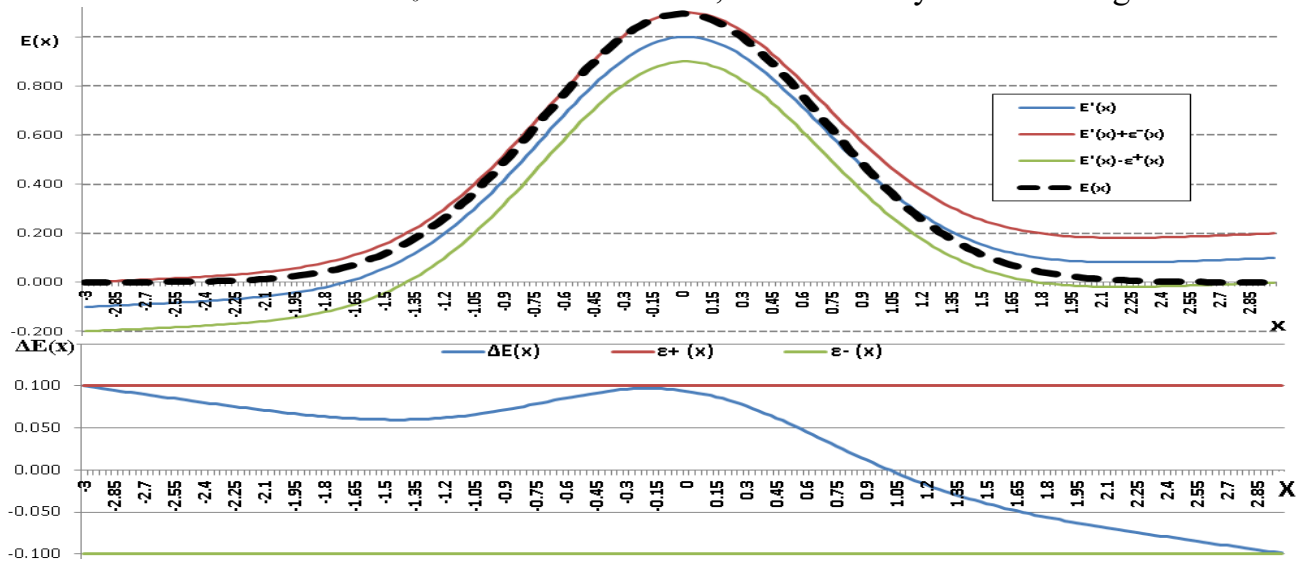

Fig. 8. Example of realization of signal $E^{\prime}(x)$ and possible boundaries $E^{\prime}(x)+\varepsilon^{-}(x)$ and $E^{\prime}(x)-\varepsilon^{+}(x)$ of a range of definition of modelling function for a case of linearly varying on an interval of observation of a background illumination from -0.1 to $0.1 . \Delta x \in[-0.942,0.942]$ with $\Delta E_{0}=0$ and $\Delta R=0$. 


\section{Conclusion}

The executed experiments have shown, that the offered methods for calculation of greatest possible and actually observable interval errors are very simple and useful to applications when noise and interferences during conducting of procedure of measurements do not manifest statistical properties. The considered concrete example of the application of the method for solving the particular problem of positioning objects by optical methods has shown that the use of the $\varepsilon$ - layer model makes it possible, without complex calculations, to analyze the influence of various factors on the accuracy of measurements and to obtain both qualitative and quantitative results.

\section{Acknowledgement}

The author expresses his deep gratitude to the leadership of the Altai State Technical University of I.I. Polzunov (AltSTU) for financial support of the publication of the article and OOO Promavtomatika for technical assistance in the preparation of the article.

\section{References}

[1] V.A. Balalaev, V.A. Slaev, A.I. Sinjakov, Potential accuracy of measurements. Scientific issue (Scientific professional unity «Professional», St.Peterburg, 2005) (in Russian)

[2] B.R. Levin, Theoretical bases of a statistical radio engineering (Soviet Radio, Moscow, 1975) (in Russian)

[3] S.E. Phalkovich, A.N. Homjakov, The statistical theory of measuring radio systems (Radio\&Communication, Moskow, 1981) (in Russian)

[4] J.R.Taylor, An Introduction to Error Analysis: The Study of Uncertainties in Physical Measurements (University Science Books, 1999)

[5] R.B. Ash, A.Cathterine, Doléans-Dade, Probability \& Measure Theory, Second Edition (Academic Press, 2000)

[6] K.B. Athreya, S.N. Lahiri, Measure Theory and Probability Theory (Springer Science + Business Media, 2006)

[7] P. Billingsley, Probability and Measure, Third Edition (John Wiley \& Sons, 1995)

[8] R.E. Moore, Interval analysis ( Englewood Cliffs. Prentice Hall, 1966)

[9] R.E. Moore, R.B.Kearfott, M.J.Cloud, Introduction to interval analysis (SIAM, Philadelphia, 2009)

[10] R.E. Moore, Methods and applications of interval analysis (SIAM, Philadelphia, 1979)

[11] O.Ye Rodionova, A.L.Pomerantsev, In: «Progress in Chemometrics Research», 43 (2005)

[12] S. Markov, K. Okumura, In: «Developments in Reliable Computing», 167 (1998)

[13] A.G. Yakunin, Scientific Pract.Conf. «Optical scanners and devices on their basis», 169 (1986) (in Russian)

[14] L.I. Suchkova, A.G. Yakunin, The bulletin of Dagestan State Technical University. Technical Sciences, 4 11(2011) (in Russian)

[15] L.I. Suchkova, A.G. Yakunin, Proc.of TSU of Control Systems and Radioelectronics 2(28), 147(2013) (in Russian)

[16] P.V. Novitskij Bases of the information theory of measuring devices (Energy, Leningrad,1968) (in Russian)

[17] P.V. Novitskij, I.A. Zograph, Estimation of errors of results of measurements (Energoatomizdat, Leningrad, 1991) (in Russian) 
[18] S. Donati, Electro-Optical Instrumentation: Sensing and Measuring with Lasers 1st Edition (Pearson Education, New Jersey, Inc.,2004)

[19] P.C.D. Hobbs, Building Electro-Optical Systems: Making It all Work (Wiley series in pure and applied optics) (John Wiley \& Sons Inc., USA, 2009)

[20] P. Fiala, T. Jirku, R. Kubasek, P. Drexler, P. Konas, PIERS ONLINE, 2, 685 (2006)

[21] S.P. Mahulikar, H.R. Sonawane, G.A. Rao, Progress in Aerospace Sciences, 43(7-8), 218 (2007)

[22] E.R. Fossum, IEEE Transactions on Electron Devices, 44, 10, 689 (1997)

[23] H.S. Wong, IEEE IEDM Technical Digest, 8.5.1(1997)

[24] M. Bom, W. Emil. Principles of Optics, 6lh edition (Cambridge University Press, reissued, 1998) 